International Journal of Current Advanced Research

ISSN: O: 2319-6475, ISSN: P: 2319 - 6505, Impact Factor: SJIF: 5.995

Available Online at www.journalijcar.org

Volume 6; Issue 3; March 2017; Page No. 2399-2404

DOI: http://dx.doi.org/10.24327/ijcar.2017.2404.0017

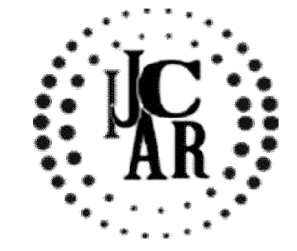

Research Article

\title{
SKIN PRICK TEST SENSITISATION PATTERNS IN CHILDREN AND ADULTS PRESENTING WITH ALLERGY SYMPTOMS IN BANGALORE - A RETROSPECTIVE ANALYSIS
}

\section{Sowmya Arudi Nagarajan*., Harsha N S and Jayalakshmi K}

People Tree Allergy Center, People Tree Hospitals, \# 2, Tumkur Road, Goraguntepalya

(next to Yeshwanthpur Industry Metro Station), Bengaluru- 560022

A R T I C L E I N F O

\section{Article History:}

Received $28^{\text {th }}$ December, 2016

Received in revised form $25^{\text {th }}$ January, 2017

Accepted $4^{\text {th }}$ February, 2017

Published online $28^{\text {th }}$ March, 2017

\section{Key words:}

Allergen, Allergy, Skin prick test, Allergic Rhinitis, Asthma

\begin{abstract}
A B S T R A C T
Objective: To demonstrate the allergic sensitisation pattern in children and adults presenting with allergy symptoms in multi-centre allergy clinics in North Bangalore observed through Skin Prick Test.

Methods: Data on sensitisation, clinical history, physical examination, and diagnosis of individuals who presented to multi-centre allergy clinics with allergic symptoms, and underwent skin prick test, was analysed retrospectively, to establish sensitisation pattern, its correlation to clinical presentation and variation in paediatric and adult population.

Results: 35 children and 46 adults underwent Skin Prick Test for their allergy workup from March to November 2016. Allergic rhinitis was commonest among adults $(60.86 \%)$ and asthma $(51.43 \%)$ in children.

Aeroallergen sensitisation was seen in $78.26 \%$ of adults and $85.7 \%$ of children. Adults were sensitised to house dust mites (Blomia tropicalis - 57.14\%, Dermatophagoides pteronyssinus \& farinae - 47.83\% \& 30.43\%), Cockroach - 29.27\%, pollens (Parthenium hysterophorus - 29.27\%), and molds (Alternaria alternata - 29.27\%, Aspergillus fumigatus - 17.07\%).

Children were sensitised to house dust mites (Dermatophagoides pteronyssinus \& farinae - $80.00 \%$ \& $68.57 \%$, Blomia tropicalis - 40.00\%), Cockroach $(17.65 \%)$ and pollens (Parthenium hysterophorus - 14.71\%).

Differences were noted in pattern of sensitisation for these allergens in the allergic rhinitis, asthma and the 'rhinitis with asthma' groups, which are discussed in the manuscript.

Conclusions: Our cohort was sensitized to common airway allergens with differences in sensitisation pattern in adult and pediatric population and in the different allergy phenotypes. Knowledge of sensitisation pattern and differences within the population enables clinicians to provide patient-centric diagnosis and treatment including pharmacotherapy and immunotherapy.
\end{abstract}

Copyright $₫ 2017$ Sowmya Arudi Nagarajan, Harsha $\mathbf{N}$ S and Jayalakshmi K. This is an open access article distributed under the Creative Commons Attribution License, which permits unrestricted use, distribution, and reproduction in any medium, provided the original work is properly cited.

\section{INTRODUCTION}

Allergic conditions like asthma, allergic rhinitis, eczema and urticaria are encountered frequntly by clinicians. $20-30 \%$ of Indians reportedly suffer from allergic conditions, attributable to rapid urbanisation and lifestyle changes. ${ }^{[1][2]}$ These IgE mediated conditions are triggered by sensitisation to common environmental allergens. [1][3] The sensitisation pattern triggering allergic reactions varies across geophraphies and over a period time. ${ }^{[4]}$ Awareness of sensitisation pattern in a particular geography enables clinicians to identify offending allergens effectively. ${ }^{[5]}$ Hence studies reporting allergen sensitisation patterns in a particular region become important.

*Corresponding author: Sowmya Arudi Nagarajan

People Tree Allergy Center, People Tree Hospitals, \# 2, Tumkur Road, Goraguntepalya (next to Yeshwanthpur Industry Metro Station), Bengaluru- 560022
Skin prick test (SPT) is considered to be the preferred diagnostic test for IgE mediated allergic sensitisation, compared to intradermal and in-vitro serum specific IgE tests. It is a widely used test, simple to perform, highly specific, relatively inexpensive, and gives immediate results that can be visually perceived by the patients. Many allergens can be tested simultaneously with very low risk of anaphylactic reaction. ${ }^{[3][6]}$

\section{MATERIALS AND METHODS}

The records of all patients presenting with allergic symptoms to multi-centric allergy clinics including a tertiary care hospital and two private practices in Bangalore were reviewed. These patients underwent Skin Prick Test in the course of their allergic evaluation from March 2016 to November 2016. The patient population that included both children $(<18$ years of age) and adults ( $>18$ years of age), 
were reviewed with respect to their clinical diagnosis and allergen sensitisation pattern. We also assessed the sensitisation pattern in each population group based on their clinical diagnosis at the time of presentation. Individuals with a history suggestive of obstructive airway disease and who reported consistent clinical response to bronchodilator therapy were classified as having asthma for the purpose of this study.

At the time of presentation, a detailed clinical history and physical examination was done as a part of clinical evaluation for each patient. The procedure and risks involved with SPT were explained and SPT was performed on consenting individuals (parental consent was obtained in case of paediatric patients). The patients were educated about the possible drug interactions with inhibitory effect on SPT and were asked to abstain from all these drugs for appropriate duration including antihistamines for at least 120 hours prior to the procedure of SPT. Antigen panel was chosen based on the details of clinical history and the relevant clinical literature. The standard skin prick test procedure, as outlined by EAACI guidelines was followed. [7] The test was performed on the volar aspect of the forearm or back using standard technique. Histamine was used for positive control and normal saline for negative control. Wheal diameter was measured using a whealometer 15 minutes after the application of the allergen extracts. Standard SPT lancets from Allergopharma (Merck- Germany) were used to give the pricks. The largest diameter of the wheal was used to determine positivity. [Image 1] Wheal of $\geq 3 \mathrm{~mm} \mathrm{(3 \textrm {mm } >}$ than negative control) in cases where there were appropriate positive histamine reactions ( $\geq 3 \mathrm{~mm}$ ) were considered, since the longest diameter is considered a better estimate of wheal surface area than the mean perpendicular diameters of a skin prick test above a certain value.

Standardized allergen extracts from Allergopharma (MerckGermany) were used for the skin prick test. Allergen extracts which were not standardized and unavailable with earlier supplier, were sourced from Creative Diagnostic Medicare Private Ltd, Navi Mumbai (CREDISOL®). However, for individual antigens, antigen sourced from the same vendor was used for all patients (eg:CREDISOL $\AA$ Cockroach allergen was used for all patients).

Microsoft Excel (Version - 2003) was used to enter and analyse the data. Descriptive statistics like frequency, percentage, and proportions were used to analyse the data. An institutional review board clearance and from individual clinicians involved in the study were obtained prior to the analysis.

The antigens tested based on clinical history included Dermatophagoides pteronyssinus (Der. P/D. pteronyssinus), Dermatophagoides farinae (Der. F/D. farinae), Blomia tropicalis (Blomia/B. tropicalis) - [house dust mites /HDM]; Alternaria alternata (Alternaria), Aspergillus fumigatus (Aspergillus), Candida albicans - [fungi]; Parthenium, Bermuda grass, Timothy grass, Ricinus communis (Ricinus/R. communis), Mugwort, Lambs Quarters, Ragweed, Eucalyptus, Kentucky grass - [pollens]; Chocolate, English plantain [food], Cat epithelium, Dog epithelium and Cockroach allergen.

\section{RESULTS}

A total of 81 individuals including 46 adults and 35 children had undergone skin prick test for various allergic conditions. Median age was 6 years in children (Range: 1year, 4 months 17 years) and 37 years in adults (Range: 21 years - 67 years). There were 23 females and 23 males among adults and 19 females and 16 males among children. [Tables $1 \& 2$ ]

Table 1 Age Distribution of the Cohort (in Years)

\begin{tabular}{cccc}
\hline & Minimum & Maximum & Median \\
\hline Children & 1.25 & 17 & 6 \\
Adults & 21 & 67 & 37 \\
\hline
\end{tabular}

Table 2 Gender Distribution of the Cohort

\begin{tabular}{cccc}
\hline & Total & Male & Female \\
\hline Children & 35 & 16 & 19 \\
Adults & 46 & 23 & 23 \\
\hline
\end{tabular}

Clinical Presentation: In the present cohort, patients presented pre-dominantly with airway allergies $(97.83 \%$ of adults and $88.6 \%$ of children)

Allergic rhinitis was the most frequent clinical condition among adults $(60.86 \%)$ followed by asthma $(21.74 \%)$ and asthma with allergic rhinitis (15.22\%).

Among children, asthma was the predominant clinical presentation (51.43\%) followed by Allergic rhinitis (25.71\%) and asthma + allergic rhinitis (11.43\%).

Urticaria with rhinitis and atopic dermatitis accounted for the rest $(2.17 \%$ in adults and $11.4 \%$ in children) [fig 1 , fig 2]
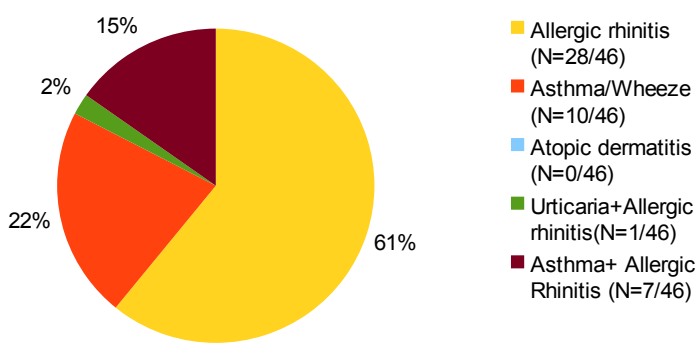

Fig 1 Clinical diagnosis at presentation among adults presenting with allergic symptoms

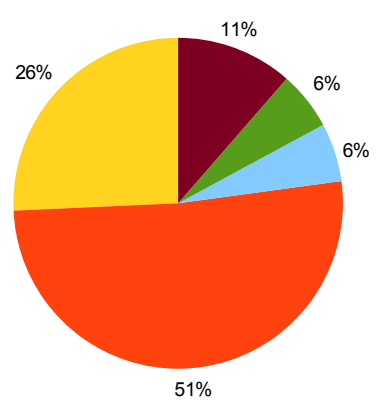

Allergic rhinitis $(\mathrm{N}=9 / 35)$

Asthma/Wheeze

$(\mathrm{N}=18 / 35)$

Atopic dermatitis $(\mathrm{N}=2 / 35)$

- Urticaria+Allergic

rhinitis $(\mathrm{N}=2 / 35)$

Asthma+ Allergic Rhinitis

$(\mathrm{N}=4 / 35)$

Fig 2 Clinical diagnosis at presentation among children presenting with allergic symptoms 
Sensitisation to aeroallergens were seen in $78.26 \%$ of adults and $85.7 \%$ of children.

Adults were predominanlty sensitised to house dust mites (Blomia - 57.14\%, Der .P - 47.83, Der .F - 30.43\%), followed by Alternaria, Parthenium and Cockroach. [fig 3]

Among children, house dust mite allergy was again the commonest (Der. P - 80.00\%, Der .F - 68.57\% and Blomia $40.00 \%$ ) followed by Cockroach (17.65\%) and Parthenium (14.71\%). None of the children tested were sensitised to food allergens or Aspergillus. [fig 4]

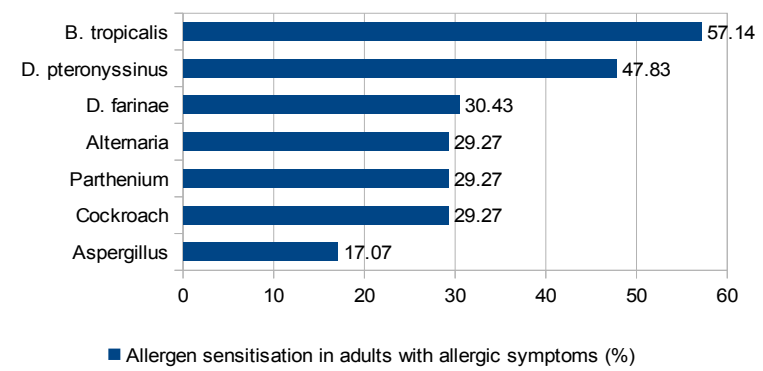

Figure 3 Allergen sensitisation in adults with allergic symptoms (\%)

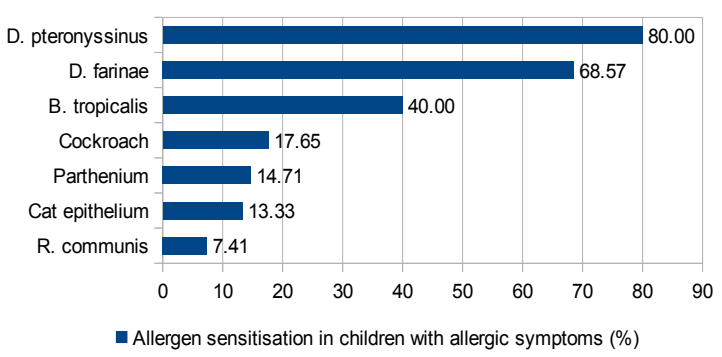

Figure 4 Allergen sensitisation in children with allergic symptoms (\%)

Among adults presenting with allergic rhinitis, dust mites (Der. P- 42.86\% and Der. F- 17.86\%), Cockroach (32\%) and Parthenium - 29.17\%) were the frequent sensitisers whereas in adults with asthma sensitivity was commonly seen for house dust mites (Der. F, Der. P and Blomia - 40\%), Alternaria (37.5\%), Parthenium and Aspergillus-33.33\%. [fig 5 and fig 6]

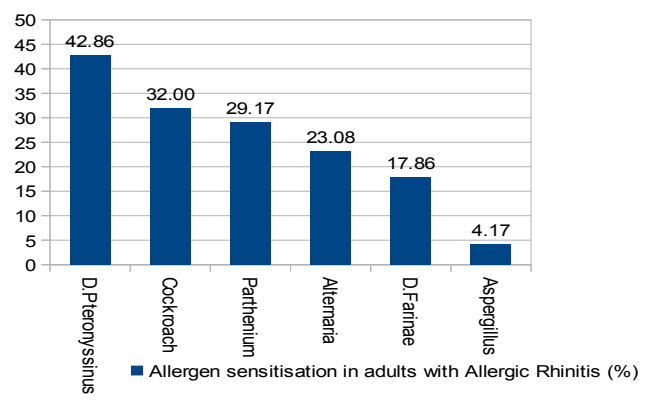

Figure 5 Allergen sensitisation in adults with Allergic Rhinitis (\%)

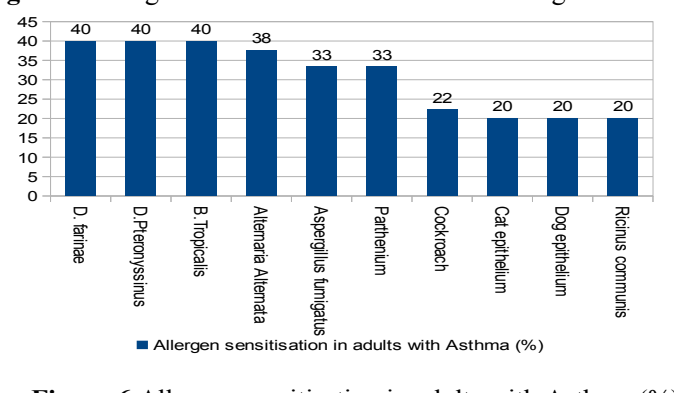

Among children with Allergic rhinitis house dust mites were the common allergens (Der. P - 77.78\%, Der. F - 66.67\% and Blomia - 33.33\%) followed by Cockroach (33.33\%) and pet dander (cat epithelium - 16.67\%). Children presenting with asthma, showed sensitisation to house dust mites (Der. P$83.33 \%$, Der. F - 77.78\%, and Blomia - 50\%), pollens (Parthenium - 23.53\% and Ricinus - 7.14\%), cat epithelium (18.75\%) and Cockroach (17.65\%). In our cohort none of the children were sensitised to Aspergillus or had allergy to the foods tested. [fig 7 and fig 8]

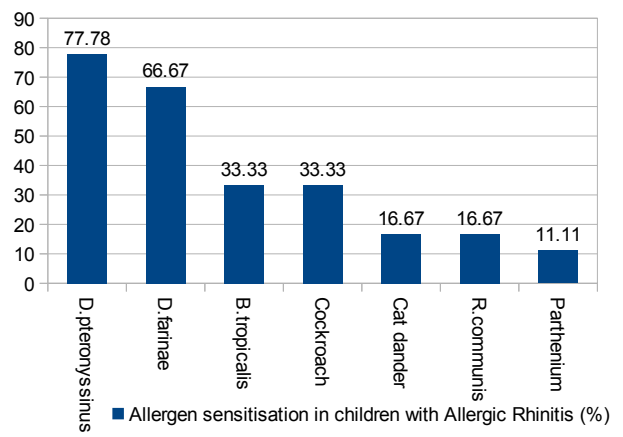

Figure 7 Allergen sensitisation in children with Allergic Rhinitis (\%)

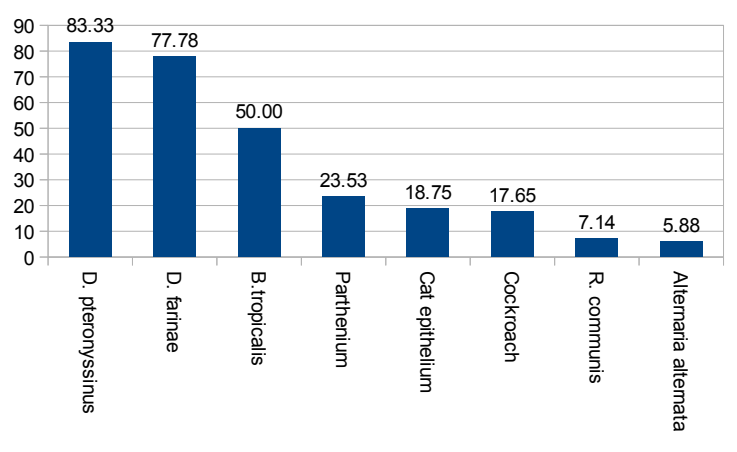

- Allergen sensitisation in children with Asthma (\%)

Figure 8 Allergen sensitisation in children with Asthma (\%)

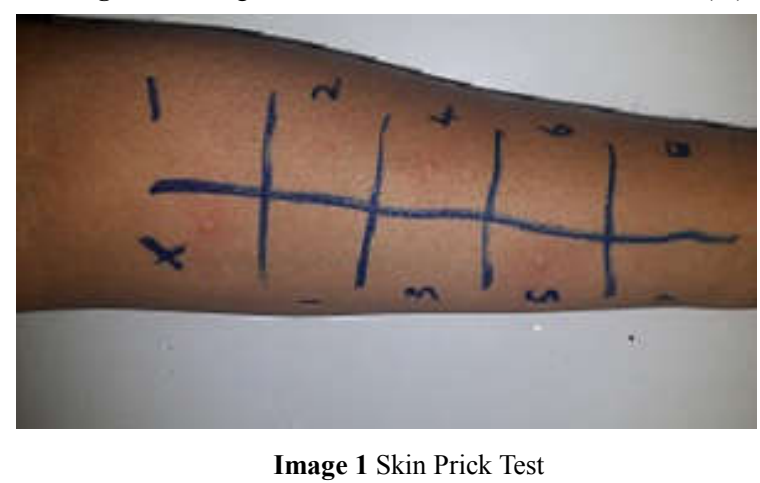

Among individuals with asthma, sensitisation to Aspergillus fumigatus was seen in $33.3 \%$ of adults and $28.57 \%$ of those with rhinitis and asthma whereas none of the children with airway allergies showed sensitisation to aspergillus. [Table 3]

Table 3 Sensitisation to Aspergillus among individuals with Asthma

\begin{tabular}{ccc}
\hline & Asthma & $\begin{array}{c}\text { Asthma + allergic } \\
\text { rhinitis }\end{array}$ \\
\hline Children & $0.00 \%$ & $0.00 \%$ \\
Adults & $33.33 \%$ & $28.57 \%$ \\
\hline
\end{tabular}




\section{DISCUSSION}

Majority of the patients in the cohort presented with symptoms consistent with airway allergies which was reflective of the relative high incidence of airway allergies in the community as with other earlier studies. ${ }^{[8][9]}$

Respiratory allergies are also found to be commonest in the community with about $20 \%$ of the population suffering from respiratory allergy symptoms in South India. ${ }^{[9]}$ A study involving a patient cohort of 3389 patients visiting an allergy centre in Bombay, India, also found that airway allergies (asthma, rhinitis or asthma with rhinitis) were commonest $(76.9 \%)$ in their cohort and asthma + rhinitis was the predominant presentation among both adults (35.5\%) and children $(43.8 \%){ }^{[8]}$ This is in contrast to our study where asthma was most common presentation among children (51\%) and allergic rhinitis among adults $(61 \%)$ and the concomitant presentation of asthma with allergic rhinitis was much lower ( $11 \%$ in children and $15 \%$ in adults). The concept of united airway is also an established proposition as it has been demonstrated that allergic rhinitis is a strong risk factor for subsequent development of concomitant asthma, with interplay between genetic and environmental factors. ${ }^{[10]}$ One of the possible explanation for the high incidence of asthma as compared to rhinitis in our pediatric cohort could probably reflect the voluntary healthcare seeking pattern in our region where parents of children with asthma were more often seeking specialist care as compared to parents of chidren with rhinits alone. This needs further validation through community level studies.

Aeroallergen sensitisation was seen in $78.26 \%$ of adults and $85.7 \%$ of children in our cohort. The rest did not show sensitsation to any of the allergens tested. Such a finding is consistent with observations in earlier studies. This could be due to several reasons namely, the individuals may be suffering from non-atopic respiratory conditions, may be sensitised to allergens not tested in the panel selected for the patient based on individual clinical history, or due to limitations of SPT. ${ }^{[11]}$ Further discussion on this aspect is beyond the scope of this article.

House Dust Mites are incriminated in the etiology of several atopic conditions including airway allergies. ${ }^{[12]}$ In our study HDM were the predominant allergens among both children (Der. P - 80.00\%, Der. F - 68.57\% and Blomia -40.00\%) and adults (Blomia - 57.14\%, Der. P - 47.83\%, Der. F -30.43\%). HDM sensitivity was commonest in the allergic rhinitis, asthma and rhinits + asthma groups.

This result is consistent with several other studies from India and Asia which have established HDM sensitisation as most commonly associated with allergic airway diseases. ${ }^{[4]}$ Most studies report HDM as a significant allergen in India. HDM sensitisation was commonest (50.94\%) among children with Asthma in eastern part of India and as high as $75.06 \%$ to Der. $\mathrm{P}$ in another study from the same region. ${ }^{[13][12]}$ A study from Bangalore on individuals with asthma showed that $49.28 \%$ were sensitive to house dust mites (Der. P and Der. F). ${ }^{[5]} \mathrm{A}$ study from Allahabad in northern India also found that sensitisation to HDM was commonest $(60 \%)$ in their cohort of patients with united airway diseases. ${ }^{[14]}$ In Mumbai (western part of India), HDM was commonest among sensitising allergens in people with atopic conditions (77.13\% to Der. F).

${ }^{[8]}$ Few exceptions like study from Kashmir (northern India), where pollens were the predominant sensitisers and study from Delhi (northern India) which showed house-fly to be the most frequent sensitiser and very low HDM sensitivity (7.8\%) have been reported. ${ }^{[1][15]}$

It is interesting to note that in our study $57 \%$ of adults and $40 \%$ of the children tested were sensitised to HDM of the Blomia tropicalis type. Not many Indian studies have reported or studied sensitisation to Blomia tropicalis. A study from Kolkatta, India in 2010 had shown that $72 \%$ of people with bronchial asthma were sensitised to Blomia comparable to Der. P - $75.06 \%$ and Der. F - $63.72 \%$ senitisations in the same cohort. ${ }^{[12]}$ Although Blomia is reported to be found fairly commonly in Asian homes, older studies have shown high prevalence of sensitisation in Vietnam and Singapore, whereas in rest of Asia Dermatophagoides species were the predominat sensitisers. ${ }^{[4]}$ Immunotherapy is an effective option for HDM allergy and testing for Blomia will enable us to identify and offer specific immunotherapy to individuals suffering from atopic sensitisation to either Dermatophagoides or Blomia variety. ${ }^{[16]}$ It may be prudent to test for prevalence of Blomia sensitivity in future studies in our country.

Sensitisation to Cockroach and Parthenium were next in order after HDM among both children (Cockroach - 17.65\%, Parthenium - 14.71\%) and adults (Cockroach - 29.27\%, Parthenium - 29.27\%) in our cohort. Sensitisation to cockroach antigens is not infrequent in atopic conditions and is particularly associated with asthma. Significant prevalence of cockroach sensitisation is reported world over including several parts of Asia and middle eastern countries. ${ }^{[17][4]}$ In our cohort, cockroach senitisation was more frequently found in the Allergic rhinits group among both adults and children and was the second common allergen in this group. Among the asthma group, parthenium, alternaria and aspergillus sensitisation was more common in adults, and parthenium and cat epithelium sensitisation was more common in children compared to sensitisation to Cockroach.

Earlier studies from India show varying levels of cockroach sensitisation in atopic individuals, highest prevalence reported at $-50.11 \%$ in Mumbai, 30\% in Allahabad, $18.3 \%$ in Delhi among children with asthma and $39.62 \%$ among children with asthma in Kolkotta. ${ }^{[8][15][13][14]}$ A recent study from Bangalore among individuals with asthma, found 3.6\% sensitisation to Cockroach. ${ }^{[5]}$ Our study showed much higher rates of sensitisation overall and in the asthma group (17.65\% in children and $22 \%$ in adults). Awareness about senstisation to cockroach antigen prevalence may help in decision on routine testing leading to better diagnosis and outcome to the patient in the future. Immunotherapy to cockroach antigen is non-standardised presently. However few trials have shown encouraging results. [17] Suggestions for asthma control strategies at the community level include a reduction in exposure to cockroach allergen. ${ }^{[18]}$ Our study reiterates that such an approach could be effective in our country given the high level of sensitisation across regions.

In our study, parthenium sensitisation was highest among pollens at $22.67 \%$, with adults showing higher sensitisation than children $(29.27 \%$ vs $14.71 \%)$. Parthenium has been 
found to be the commonest sensitiser among pollens in other studies as well involving individuals with airway allergies (Bombay - western part of India - $17.33 \%$, Bangalore southern part of India $-20.86 \%$ of all pollen sensitisations and Allahabad - Northern part of India - 26.66\%). ${ }^{[8][5][14]}$

Sensitisation to Aspergillus fumigatus is considered to be an important factor precipitating airway allergic disorders in atopic individuals and associated with increased disease severity in these individuals. ${ }^{[19]}$ Global Initiative for Asthma (GINA) - 2016 also mentions that in patients at risk of developing asthma, visible mold and mold odor are associated with increased risk of developing asthma. ${ }^{[20]}$ Across India reported sensitisation to aspergillus fumigatus varied from $4.32 \%$ in Bangalore to $22.28 \%$ in Kolkotta among patients with asthma. ${ }^{[5][12]}$ In Delhi $14.4 \%$ of children with asthma showed sensitisation to Aspergillus Tamari. ${ }^{[15]}$ In our cohort, $17.07 \%$ of adults were sensitised to aspergillus fumigatus. Among asthma subgroup in adults, sensitisation was 33\%. Surprisingly, none of the children were sensitised to aspergillus. Further prospective studies will be needed to adequately establish the aspergillus sensitisation pattern and any significant variation among children and adults in our geographic region.

Adults with allergic rhinits were more frequently sensitised to parthenium than molds (alternaria and aspergillus), whereas those with asthma were more often sensitised to molds (alternaria and aspergillus) than parthenium.

In our study children with a probable history of food allergy, who were tested with the corresponding allergens in the SPT did not have sensitivity to the tested allergen. Unnecessary diet restriction should not be advised especially in children without evidence of sensitisation.

Limitations: Our study was a retrospective chart review and hence all patients were not tested with the same allergen panel. Allergens chosen were not extensive but only those allergens that were relevant to the clinical history of the patient and commonly reported allergenic antigens relevant to the patients' geographic/topographic location were tested and hence may not be able to capture any new emergent allergen sensitisations.

However the clinical history based approach in antigen selection is the recommended approach in selecting antigens for SPT in clinical practice. ${ }^{[21][22]}$ Therefore our observations are likely to have good clinical correlation and represent the sensitisation patterns observed in everyday practices in our geographical area.

\section{CONCLUSIONS}

Allergic disorders remain a major health concern due to its morbidity and its relevant health costs. Diagnosis of allergy and its clinical implications may vary over many studies worldwide, but consensus has emerged about possible etiological association of aeroallergens with airway allergies. Local allergen sensitisation studies are of relevance in atopic patient education. Among the allergens that is frequently associated with airway allergies include house dust mites, molds, cockroach, pet dander and pollens, a pattern that was seen in our patient cohort also, in varying rates among children and adults and in different airway allergic phenotypes. A detailed knowledge about the relevant allergens and causative factors is essential to effectively diagnose, treat and prevent allergic disorders, especially airway allergic disorders. This exercise will help in patient centric individualised treatment which can include appropriate allergen avoidance measures, immunotherapy and probably help reduce the need for medical therapy. Food restriction must be avoided unless sufficient clinical collaboration with history and demonstration of relevant $\operatorname{IgE}$ sensitisation is present.

\section{References}

1. Prasad R, Kumar R. Allergy situation in India what is being done. Indian J Chest Dis Allied Sci, 2013 Jan; 55(1): 7-8.

2. SF Bloomfield, R Stanwell-Smith, RWR CrevelJ Pickup. Too clean, or not too clean: the Hygiene Hypothesis and home hygiene. Clin Exp Allergy. 2006 Apr; 36(4): 402-425.

3. Sinclair J, Brothers S, Jackson P et al. IgE-mediated food allergy-diagnosis and management in New Zealand children. $N$ Z Med J. 2013 Aug 16;126(1380):57-67.

4. Tham EH, Lee AJ, Bever HV.Aeroallergen sensitization and allergic disease phenotypes in Asia. Asian Pac J Allergy Immunol. 2016 Sep;34(3):181-189

5. Gowda G, Nagaraj C, Parasuramalu B G, Huliraj $\mathrm{N}$. Aeroallergen sensitivity among patients suffering from bronchial asthma in Bangalore. Int $J$ Health \& Allied Sci. 2013 Dec; 2(4): 237-241

6. Cantani A, Micera M.Can skin prick tests provoke severe allergic reactions? Eur Rev Med Pharmacol Sci. 2000 Sep-Dec;4 (5-6):145-8.

7. Heinzerling L, Mari A, Bergmann $\mathrm{KC}$ et al. The skin prick test - European standards. Clin Transl Allergy. 2013 Feb 1;3(1):3. doi: 10.1186/2045-7022-3-3.

8. Shaikh WA, Shaikh SW. Allergies in India: an analysis of 3389 patients attending an allergy clinic in Mumbai, India. J Indian Med Assoc. 2008 Apr;106(4):220, 222, 224 passim

9. Nitin J, Palagani R, Shradha NH. et al. Prevalence, severity and risk factors of allergic disorders among people in south India. African Health Sciences. 2016 Mar;16(1): 201-209.

10. Togias A. Rhinitis and asthma: evidence for respiratory system integration. J Allergy Clin Immunol. 2003 Jun;111(6):1171-83; quiz 1184.

11. Rasool R, Shera IA, Nissar S, et al. Role of Skin Prick Test in Allergic Disorders: A Prospective Study in Kashmiri Population in Light of Review. Indian $J$ Dermatol. 2013 Jan-Feb; 58(1): 12-17.

12. Podder S, Gupta SK, Saha GK, Incrimination of Blomia tropicalis as a Potent Allergen in House Dust and Its Role in Allergic Asthma in Kolkata Metropolis, India. World Allergy Organ J. 2010 May; 3(5): 182187

13. Dey S, Chakraborty T, Prevalence study of common environmental allergens in children with asthma and allergic rhinitis in Kolkata: A hospital-based study. Indian J Child Health. 2016 Jul- Sep; 3(3): 225-229

14. Mishra VD, Mahmood T, Mishra JK. Identification of common allergens for united airway disease by skin prick test. Indian $J$ Allergy Asthma Immunol 2016;30:76-9 
15. Raj D, Lodha R, Pandey A, Aeroallergen sensitization in childhood asthmatics in northern India. Indian Pediatrics. 2013 Dec; 50(12):1113-8

16. Jeevarathnum AC, van Niekerk A, Green RJ et al. Prevalence of Blomia tropicalis allergy in two regions of South Africa. S Afr Med J. 2015 Sep 21;105(7):5679

17. Pomes A, Arruda LK, Investigating cockroach allergens: aiming to improve diagnosis and treatment of cockroach allergic patients. Methods. 2014 March 1; 66(1): 75-85

18. Lombardi C, Savi E, Ridolo E. et al. Is allergic sensitization relevant in severe asthma? Which allergens may be culprits? World Allergy Organization Journal (2017) 10:2
19. Agarwal R, Gupta D. Severe asthma and fungi: current evidence. Med Mycol. 2011 Apr;49 Suppl 1:S150-7

20. Quansah R, Jaakkola MS, Hugg T.T et al. Residential Dampness and Molds and the Risk of Developing Asthma: A Systematic Review and Meta-Analysis. Plos One.2012;7(11):e47526.

21. Allergy diagnostic testing: an updated practice parameter. Ann Allergy Asthma Immunol. 2008 Mar;100(3 Suppl 3):S1-148.

22. Wallace DV, Bahna SL, Goldstein S, American Academy of Allergy, Asthma \& Immunology Work Group Report: allergy diagnosis in clinical practice. $J$ Allergy Clin Immunol. 2007 Oct;120(4):967-9. Epub 2007 Jul 16.

\section{Please cite this article in press as:}

Sowmya Arudi Nagarajan., Harsha N S and Jayalakshmi K (2017) Skin Prick Test Sensitisation Patterns In Children And Adults Presenting With Allergy Symptoms In Bangalore - A Retrospective Analysis, International Journal of Current

Advanced Research, 6(3), pp. 2399-2404.

http://dx.doi.org/10.24327/ijcar.2017. 2404.0017

$* * * * * * *$ 